\title{
EDITORIAL AND COMMENT Is Mortality Risk Reduced in Overweight or Obese Diabetics?
}

\author{
Gang Hu, MD, PhD and Steven B. Heymsfield, MD \\ Pennington Biomedical Research Center, Baton Rouge, LA, USA.
}

J Gen Intern Med 29(1):3-4

DOI: $10.1007 / \mathrm{s} 11606-013-2608-9$

( ) Society of General Internal Medicine 2013

$\mathrm{D}$ iabetes is considered a 21 st century epidemic, affecting approximately 26 million individuals in the U.S. and costing \$245 billion. ${ }^{1}$ Overweight and obesity are also important public health problems. Two in three US adults are currently classified as overweight (body mass index [BMI] $25.0-29.9 \mathrm{~kg} / \mathrm{m}^{2}$ ) or obese (BMI $\left.\geq 30 \mathrm{~kg} / \mathrm{m}^{2}\right)$, and more than one-third of US adults are obese. ${ }^{2}$

A sedentary lifestyle and unhealthy dietary habits contribute to obesity ${ }^{2}$ and diabetes. About $45-65 \%$ patients with type 2 diabetes are obese in the US. ${ }^{3}$ However, the association between BMI and mortality is unclear among patients with type 2 diabetes. To date, studies have reported inverse associations, ${ }^{3}$ positive associations, ${ }^{4} \mathrm{U}$-shaped associations, ${ }^{5}$ or no associations between BMI and mortality ${ }^{6}$ among patients with type 2 diabetes. The reasons for the difference in associations across studies are not clear, although the study samples differed at baseline by mean BMI, health status, ethnicity, and duration of diabetes.

In this issue of JGIM, Jackson and colleagues ${ }^{7}$ assess the association of BMI with the risk of total mortality among 34,805 never smokers (2,035 with diabetes and 32,770 without diabetes) from the 1997-2002 National Health Interview Survey, and show that BMI is positively associated with mortality in adults without diabetes, but inversely associated with mortality among participants with diabetes. The relationships observed in this study are strong, graded, and independent of smoking, cardiovascular disease (CVD), cancer, and a range of other potential confounders including age, marital status, leisure-time physical activity, alcohol consumption, poor income, and self-reported general health status.

The study by Jackson et al. provides important information on the "obesity paradox," which describes the inverse association between excess adiposity as defined by BMI and mortality. Another recent analysis by Carnethon et al. pooled five longitudinal studies and found that patients who are normal weight at the time of incident diabetes have a higher mortality risk than those who are overweight or obese. ${ }^{3}$ The authors suggest that "metabolically obese" normal-weight (MONW) diabetics may harbor an underlying illness that

Published online September 4, 2013 predisposes to mortality. ${ }^{8}$ Despite having a normal BMI, these individuals have hyperinsulinemia, insulin resistance, and dyslipidemia, all of which predispose to the risk of premature death. ${ }^{8}$ Individuals with MONW are becoming more common in the US, with $23.5 \%$ of normal-weight US adults now considered metabolically "abnormal".

Three potential methodological concerns should be considered when assessing the associations between obesity and health outcomes. The most serious of these is reverse causation associated with total mortality. People with a history of CVD or cancer frequently lose weight, and may thus be included at their reduced BMI in the "normal weight" population, which then spuriously increases the estimated risk of death. Most previous studies did not exclude patients with a history of CVD and cancer when they assessed the association between BMI and the risk of mortality among individuals with type 2 diabetes. ${ }^{4-6}$ The analysis by Jackson et al. ${ }^{7}$ excluded individuals who reported a prior history of cancer and/or coronary heart disease, which can minimize the influence of reverse causation.

The second major concern is that confounding factors may distort the association between body weight and mortality. Smoking is a particularly important factor, because smokers tend to weigh less and to have much higher mortality rates than nonsmokers. In most previous studies, smoking status was considered a confounding factor in multivariate models, ${ }^{4-6}$ which if accounted for, could influence the effect of smoking on both weight and mortality. The most satisfactory way to deal with the presence of smokers in a study database is to restrict the analysis to subjects who have never smoked, as did Jackson et al. and other investigators. ${ }^{3,5,7}$ However, many previous studies had small samples, and thus lacked adequate statistical power when the analysis was limited to those who have never smoked. ${ }^{4,6}$

The third methodological concern in some analyses between body weight and mortality is that the physiologic effects of excess fatness, such as hypertension, diabetes, and dyslipidemia, were controlled statistically, thus artificially removing some of the effects of being overweight. Most of the studies assessing the association of BMI and morality 
risk among individuals with type 2 diabetes have taken this potential over-adjustment for weight-related risk factors into consideration.

Thus the report by Jackson and colleagues ${ }^{7}$ is one of the best studies to control for three potential methodological problems in assessing the association of BMI with morality risk among individuals with type 2 diabetes. However, as commented on by the authors, the study had several limitations, including selfreported height, weight, and diabetes diagnosis, that should be considered when interpreting the overall results.

A frequently voiced concern is that BMI has limitations as a measure of adiposity. BMI does not account for sex, race, age, and fitness differences in fat mass even at the same body weight. Waist circumference is recommended as an additional surrogate marker of the health risks associated with adiposity and adipose tissue distribution. Only two previous studies have assessed the association of waist circumference with mortality risk among patients with type 2 diabetes. The first study, by Carnethon et al. was a pooling analysis of five longitudinal studies that indicated that for each standard deviation (SD) increase in waist circumference, there is an $18 \%(95 \% \mathrm{CI}$ 1.06-1.31) increase in total mortality risk among individuals with type 2 diabetes. ${ }^{3}$ The second report, The Pennington Center Longitudinal Study, found that for each SD increase in waist circumference $(15 \mathrm{~cm})$ and BMI $\left(7 \mathrm{~kg} / \mathrm{m}^{2}\right)$, there was an associated $41 \%$ (95 \% CI 1.15-1.73) and $32 \%$ (95 \% CI $1.07-$ 1.64) increase in total mortality risk among individuals with type 2 diabetes. More studies on other markers of adipose tissue distribution, such as other body circumferences, skinfold thicknesses, and imaging-derived measures, with the risk of mortality among patients with type 2 diabetes are needed.

The study by Jackson et al. ${ }^{7}$ indicates that mortality increases with increasing BMI in adults without diabetes, but decreases with increasing BMI among their counterparts with diabetes. Their finding in diabetic patients might be of particular interest to developers of practice guidelines for the American Diabetes Association, and might also support the recent finding from the Look AHEAD (Action for Health in Diabetes) study. ${ }^{10}$ The Look AHEAD study findings indicated that an intensive lifestyle intervention produces greater reductions in body weight and $\mathrm{HbAlc}$ and greater initial improvements in fitness and all CVD risk factors, but does not reduce the rate of CVD morbidity and mortality in overweight or obese adults with type 2 diabetes. ${ }^{10}$ Thus a need still exists to evaluate the long-term effects of weight loss on CVD risk among overweight or obese adults with type 2 diabetes. Not all patients classified as being overweight or obese, particularly those with chronic diseases, can be assumed to require weight loss treatment. Establishing BMI is only the first step toward a more comprehensive risk evaluation.

Corresponding Author: Steven B. Heymsfield, MD; Pennington Biomedical Research Center, 6400 Perkins Road, Baton Rouge, LA 70808, USA (e-mail: Steven.heymsfield@pbrc.edu).

\section{REFERENCES}

1. American DA. Economic costs of diabetes in the U.S. in 2012. Diabetes Care. 2013;36(4):1033-46.

2. Flegal KM, Carroll MD, Ogden CL, Curtin LR. Prevalence and trends in obesity among US adults, 1999-2008. JAMA. 2010;303(3):235-41.

3. Carnethon MR, De Chavez PJ, Biggs ML, et al. Association of weight status with mortality in adults with incident diabetes. JAMA. 2012;308(6):581-90.

4. Hu G, Jousilahti P, Barengo NC, Qiao Q, Lakka TA, Tuomilehto J. Physical activity, cardiovascular risk factors, and mortality among Finnish adults with diabetes. Diabetes Care. 2005;28(4):799-805.

5. Khalangot M, Tronko M, Kravchenko V, Kulchinska J, Hu G. Body mass index and the risk of total and cardiovascular mortality among patients with type 2 diabetes: a large prospective study in Ukraine. Heart. 2009;95(6):454-60.

6. McEwen LN, Karter AJ, Waitzfelder BE, et al. Predictors of mortality over 8 years in type 2 diabetic patients: Translating Research Into Action for Diabetes (TRIAD). Diabetes Care. 2012;35(6):1301-9.

7. Jackson CL, Yeh H, Szklo M, et al. Body-mass index and all-cause mortality in US adults with and without diabetes. J Gen Intern Med. 2013. doi:10.1007/s11606-013-2553-7.

8. Conus F, Rabasa-Lhoret R, Peronnet F. Characteristics of metabolically obese normal-weight (MONW) subjects. Appl Physiol Nutr Metab Physiol Appl Nutr Metab. 2007;32(1):4-12.

9. Wildman RP, Muntner P, Reynolds $\mathbf{K}$, et al. The obese without cardiometabolic risk factor clustering and the normal weight with cardiometabolic risk factor clustering: prevalence and correlates of 2 phenotypes among the US population (NHANES 1999-2004). Arch Intern Med. 2008;168(15):1617-24.

10. Look ARG, Wing RR, Bolin P, et al. Cardiovascular effects of intensive lifestyle intervention in type 2 diabetes. N Engl J Med. 2013;369(2):145-54. 